\title{
Temporal discrimination as a function of total presentation time
}

\author{
DONALD H. THOR and HERMAN H. SPITZ, E. R. Johnstone \\ Training and Research Center, Bordentown, N.J. 08505
}

Two triangles $(\Delta$ and $\nabla$ ) were presented successively at the same spatial locus for binocular viewing. Manipulation of stimulus duration and interstimulus interval revealed that accuracy in discriminating order is dependent upon total time $\left(S_{1}+I S I+S_{2}\right)$ and that the discrimination threshold $175 \%$ correct) is approximately $100 \mathrm{msec}$.

A classic problem in psychology, somewhat analogous to the early problems in physics regarding the nature of energy emission, is concerned with whether psychological time is continuous or consists of discrete intervals (James, 1890). Investigators have attempted to assess discrete "psychological moments" by measuring the threshold for visual discrimination of temporal order (Stroud, 1955; White, 1963).

Efron (1967) has stated that a crucial parameter for veridical perception of temporal successiveness is the processing time of the first stimulus. The first stimulus must be unimpeded for "appreciably more than 20 milliseconds [p. 723]" before presentation of the second stimulus in order for the events to be perceived as successive. If the second stimulus occurs within this processing period, it is incorporated and perceived as simultaneous. The threshold for successivity discrimination delimits the duration of the processing period, which Efron suggests is "no longer than 60-70 milliseconds [p. 726]."

According to Fraisse (1966), on the other hand, the important duration is not the processing time of the first stimulus, but rather the total time of the two successive stimuli, including the interstimulus interval. By his determination, the psychological moment is said to be $80-120 \mathrm{msec}$. That is, until the total time reaches approximately $100 \mathrm{msec}$, successive events are perceived as simultaneous.

A critical determination is whether or not the percentage of correct responses is the same when varying combinations of stimulus duration and interstimulus interval sum to the same total time. If the total time concept is correct, the specific combination of stimulus durations and ISI should be less important than their summated duration in discriminating temporal order.

\section{METHOD}

Two stimuli were presented by use of a three-channel Scientific Prototype, Model GB tachistoscope allowing three consecutive presentation-times with accuracy of better than $2 \%$. Each stimulus was a triangle with $60-\mathrm{mm}$ sides and a $75-\mathrm{mm}$ base, with lines $6 \mathrm{~mm}$ wide made by applying matte black, pressure sensitive tape (Chart-Pak) on a white $127 \times 178 \mathrm{~mm}$ card. One triangle was placed with apex up and the other with apex down when positioned in the far alleys of the tachistoscope. At very brief exposure times and very short interstimulus intervals a composite star figure was observed.

Luminance was adjusted to $6 \mathrm{ft}-\mathrm{L}$ for each field, as measured from the eyepiece, by an Elwood Foto-Meter, Model Z-4. Pre- and postexposure fields were of the same luminance and contained a small fixation cross of lightly pencilled lines on a white background. From the eyepiece, each triangle subtended $2 \mathrm{deg} 47 \mathrm{~min}$ of visual angle in the vertical dimension and $3 \mathrm{deg} 29 \mathrm{~min}$ in the horizontal dimension. When the figures fused as a star, the vertical dimension was $3 \mathrm{deg} 43 \mathrm{~min}$ and the horizontal $3 \mathrm{deg} 29 \mathrm{~min}$.

Duration was identical for the first and second stimulus during any one trial, with exposures of 10,30, 50,70, and $90 \mathrm{msec}$. Each order (apex up-blank-apex down; apex down-blank-apex up) was presented once to each $O$ at each exposure duration and at each interstimulus interval. The resulting $\mathbf{5 0}$ time combinations (five stimulus durations by five interstimulus intervals by two orders of presentation) were randomized for presentation by the method of constant stimuli.

Eleven adults, six men and five women, served as Os. Their ages ranged from 21 to 49 years with a mean of 34 years. They were instructed to report which stimulus (apex up or down) came on last or if the images were fused. Viewing was binocular throughout.

\section{RESULTS AND DISCUSSION}

Figure 1 shows the percentage of correct responses for all Os at each total time. Clearly, there are no significant differences for data points representing different combinations of stimulus duration and interstimulus interval, as long as the total times are comparable. A curve is generated which has its maximum inflection between 50 and $150 \mathrm{msec}$. The upper difference threshold (75\% correct) ${ }^{1}$ is between 90 and $110 \mathrm{msec}$. These results corroborate the findings of Fraisse.

Those points on the curve where $S_{1}$ and ISI total at least $60 \mathrm{msec}$ and the total time is less than $100 \mathrm{msec}$ are critical for comparing the predictions made from the total time concept $\left(S_{1}+I S I+S_{2}=100 \mathrm{msec}\right)$ as opposed to the processing time concept $\left(S_{1}+I S I=60 \mathrm{msec}\right)$. There are three such points $(10-50-10,10-70-10,30-30-30)$, and the percentages of veridical responses are, respectively, $45 \%, 64 \%$, and $68 \%$. None of these percentages reaches threshold, but as the total time approaches $100 \mathrm{msec}$, correct responses approach $75 \%$. Also, at the two points where the processing times for the first stimulus are equal $[(10-50)-10]$ and $[(30-30)-30]$ but the total times differ (70 and 90), the longer total time tends to generate a greater number of correct responses $(p<.10)$ These results also support the total time concept.

It would be presumptuous to claim any single experiment

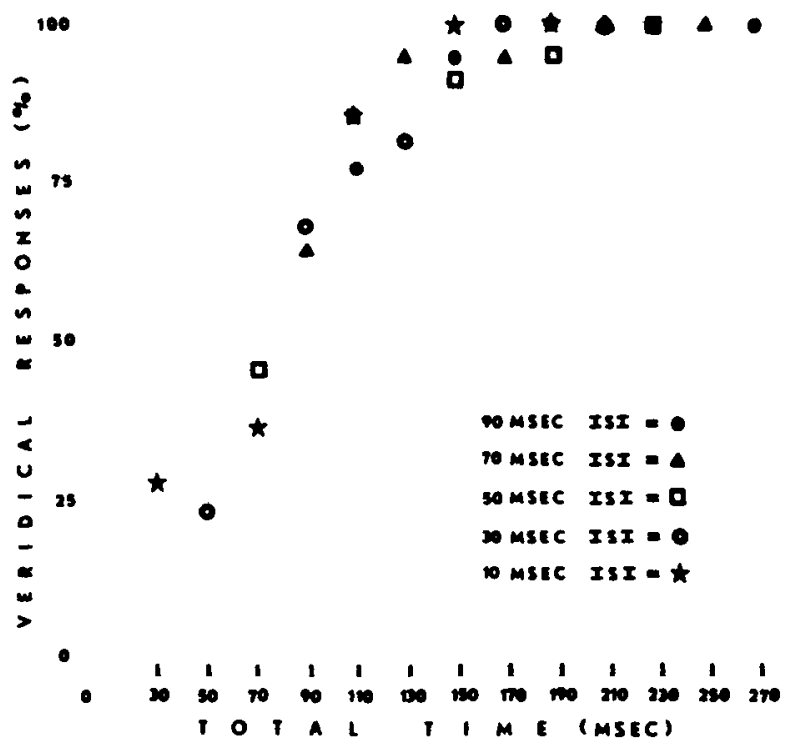

Fig. 1. Per cent correct response as a function of total time $\left(S_{1}+I S I+\right.$ $S_{2}$ ). Duration $S_{1}=S_{2}$ at all points. 
capable of determining the existence of psychological moments. Further inquiry must certainly include some resolution of the definition problems surrounding the concept of a processing period or time required for a percept to occur.

For the specific conditions of this experiment, a lawful curve was generated which supports the total time concept in the measurement of temporal discrimination. If the total time threshold for perception of order defines the duration of the psychological moment, then the present results support the suggestion that the average duration of the psychological moment in visual perception is approximately $100 \mathrm{msec}$. REFERENCES

EFRON, R. The duration of the present. Annals of the New York Academy of Sciences, 1967, 138, 713-729.
FRAISSE, P. Visual perceptive simultaneity and masking of letters successively presented. Perception \& Psychophysics, 1966, 1, 285-287. JAMES, W. The principles of psychology. Vol. 1. New York: Dover, 1950. Pp. 605-642. Originally published in 1890 by Henry Holt \& Co. STROUD, J. M. The fine structure of psychological time. In $H$. Quastler (Ed.), Information theory in psychology. Glencoe, Illinois: The Free Press, 1955. Pp. 174-205.

WHITE, C. T. Temporal numerosity and the psychological unit of duration. Psychological Monographs: General \& Applied, 1963, 77, No. 575 .

\section{NOTE}

1. Eighty six per cent of the incorrect responses were "together" (fused image) responses, so that essentially there were only two response categories. 